Nagumo, $M$.

Osaka Math. J.

15 (1963), 1-10.

\title{
PERTURBATION AND DEGENERATION OF EVOLUTIONAL EQUATIONS IN BANACH SPACES
}

Dedicated to Professor K. Shoda on his sixtieth birthday

By

Mitio NAGUMO

\section{§ 1. Completely well posed evolutional equations}

Let $E$ be a Banach space and $E_{1}$ be another Banach space such that $E \subset E_{1}$ and the embeding of $E$ into $E_{1}$ is continuous. Let $A(t)$ be a continuous linear mapping of $E$ into $E_{1}$ for every fixed $t$ in the real interval $[a, b]$ such that $A(t) u$ is a continuous function on $[a, b]$ into $E_{1}$ for every fixed $u \in E$. Then we can easily see, $A(t) u(t)$ is continuous on $[a, b]$ into $E_{1}$, if $u(t)$ is continuous on $[a, b]$ into $E$.

As an $E$-solution in $[a, b]$ of the evolutional equation

$$
\partial_{t} u=A(t) u+f(t) \quad\left(\partial_{t}=\frac{d}{d t}\right),
$$

where $f(t)$ is an $E$-continuous function on $[a, b]^{1)}$, we understand an $E$-continuous function $u=u(t)$ on $[a, b]$ such that the strong derivative $\partial_{t} u=\lim _{h \rightarrow 0} h^{-1}\{u(t+h)-u(t)\}$ exists in $E_{1}$ for $t \in[a, b]$ and the equation (0) is fulfilled in $E_{1}$ for $t \in[a, b]$.

The equation (0) is said to be $E$-well posed (or simply well posed) in $[a, b]$ when for any $\phi \in E$ there exists one and only one $E$-solution $u=u(t)$ of $(0)$ with the initial value $u(a)=\varphi$. We say that the equation (0) is completely E-well posed in $[a, b]$ when $(0)$ is $E$-well posed for any closed subinterval of $[a, b]$ and the solution $u=u(t, s, \varphi)$ of $(0)$ with the initial value $u(s)=\rho(a \leq s \leq b)$ is a continuous function of $(t, s, \varphi)$ for $a \leq s \leq t \leq b, \varphi \in E$. If $(0)$ is (completely) $E$-well posed in $[a, b]$ then the associated homogeneous equation

$$
\partial_{t} u=A(t) u
$$

1) $f(t)$ is said to be $E$-continuous on $[a, b]$ when $f(t)$ is continuous on $[a, b]$ into $E$. 
is also (completely) $E$-well posed in $[a, b]$. When (1) is completely well posed in $[a, b]$ then the solution of (1) with the initial condition $u(s)=\boldsymbol{p}$ $(s \in[a, b])$ can be written in the form

$$
u=U(t, s) \mathcal{P}
$$

where $U(t, s)$ is a continuous linear operator on $E$ into $E$ for $a \leq s \leq t \leq b$ with the following properties:

1) $U(t, s) \phi$ is continuous on $a \leq s \leq t \leq b, \mathcal{P} \in E$ into $E$,

2) $U(s, s)=1$ (identity) for $s \in[a, b]$

3) $U(t, \sigma) U(\sigma, s)=U(t, s)$ for $a \leq s \leq \sigma \leq t \leq b$,

4) $\partial_{t} U(t, s) \phi=A(t) U(t, s) \mathcal{P}$ in $E_{1}$ for $a \leq s \leq t \leq b, \mathcal{P} \in E$.

Such an operator $U(t, s)$ is called the fundamental solution of (1).

Especially when $A(t)$ does not depend on $t: A(t)=A,(1)$ is completely $E$-well posed in any finite interval $[a, b]$, if and only if (1) is simply $E$-well posed in some finite interval. For, the fundamental solution of (1) has the form $U=U(t-s)$. In this case, restricting the domain of $A$ to such a set of $u$ that $A u \in E, A$ is the infinitesimal generator of the one-parameter semi-group $\{U(t)\}_{t \geq 0}$, since $U(t+s)=U(s) U(t)$ for $s, t \geq 0$. Conversely, if $A$ is the infinitesimal generator of a one-parameter semi-group $\{U(t)\}_{t \geq 0}$, then extending the domain of $A$ on $E$ in such a way that the range of $A$ will be contained in $E_{1}$ as given in Remark 1, we obtain a completely $E$-well posed equation (1) with $A(t)=A$, for any finite interval, with the fundamental solution $U(t-s)=\exp ((t-s) A)$.

We can easily obtain the following:

Theorem 1. If the homogeneous equation (1) is completely E-well posed in $[a, b]$, and $f(t)$ is E-continuous on $[a, b]$, then the inhomogenous equation (0) is also completely E-well posed in $[a, b]$ and any solution of (0) satisfies

$$
u(t)=U(t, s) u(s)+\int_{s}^{t} U(t, \sigma) f(\sigma) d \sigma \quad \text { for } \quad a \leq s \leq t \leq b
$$

with the fundamental solution $U(t, s)$ of $(1)$.

REMARK 1. Let $A(t)$ be a pre-closed linear operator in $E$ for every $t$, and let there exists a closed operator $A_{0}$ with a domain in $E$ such that for the adjoint operators $A^{*}(t)$ and $A_{0}^{*}$ of $A(t)$ and $A_{0}$ resp. we have

$$
\left\|A^{*}(t) u^{\prime}\right\| \leq\left\|A_{0}^{*} u^{\prime}\right\|+\left\|u^{\prime}\right\| \text { for } u^{\prime} \in \mathscr{D}^{*}=\mathscr{D}\left(A_{0}^{*}\right) .
$$

Then, defining a new norm of $u \in E$ by 


$$
\|\| u \|\left|=\sup _{u^{\prime} \in \mathscr{D}^{*}}\right|\left\langle u, u^{\prime}\right\rangle \mid\left(\left\|A_{0}^{*} u^{\prime}\right\|+\left\|u^{\prime}\right\|\right)^{-1},
$$

we get $\|u\|\|\leq\| u \|$. Hence, denoting by $E_{1}$ the completion of $E$ with respect to the new norm, we obtain that the injection of $E$ into $E_{1}$ is continuous and the extension of $A(t)$ on $E$ is continuous on $E$ into $E_{1}$. Cf [1].

On the other hand, if there exists a closed operator $A_{0}$ with a domain $\mathscr{D}$ dense in $E$ such that

$$
\|A(t) u\| \leq\left\|A_{0} u\right\|+\|u\| \text { for } u \in \mathscr{D},
$$

then difining a new norm of $u \in \mathscr{D}$ by

$$
\|\| u\|=\| A_{0} u\|+\| u \|
$$

the vector space $\mathscr{D}$ becomes a Banach space $E_{0}$ with the new norm, such that the injection of $E_{0}$ into $E$ is continuous and $A(t)$ is continuous on $E_{0}$ into $E$ for every $t \in[a, b]$.

\section{$\S 2$. Stability of solutions of evolutional equations containing a parameter} $\varepsilon \geq 0$ :

Now we consider an evolutional equation containing a parameter $(1)_{\varepsilon}$

$$
\partial_{t} u=A_{\varepsilon}(t) u+f_{\varepsilon}(t) .
$$

Let $u=u_{0}(t)$ be an $E$-solution of $(1)_{0}$ in $[a, b]$ for $\varepsilon=0 . \quad u=u_{0}(t)$ is said to be completely $E$-stable in $[a, b]$ with respect to the equation $(1)_{\varepsilon}$ for $\varepsilon \rightarrow 0$, when the following condition is fulfilled: For any $\delta>0$ there evists $\eta(\delta)>0$ such that, if $0<\varepsilon<\eta(\delta)$, any $E$-solution $u=u_{\varepsilon}(t)$ of $(1)_{\varepsilon}$ on $[s, b]$ for any $s \in[a, b]$ with $\left\|u_{\varepsilon}(s)-u_{0}(s)\right\|<\eta(\delta)$ satisfies the inequality

$$
\left\|u_{\varepsilon}(t)-u_{0}(t)\right\|<\delta \text { for } s \leq t \leq b .
$$

Lemma 1. Let the equation $(1)_{\varepsilon}$ be completely $E$-well posed in $[a, b]$ for $\varepsilon>0$. If an E-solution $u=u_{0}(t)$ of $(1)_{0}$ is completely E-stable in $[a, b]$ for $\varepsilon \rightarrow 0$ with respect to $(1)_{\varepsilon}$, then the fundamental solution $U_{\varepsilon}(t, s)$ of the associated homogeneous equation of $(1)_{\varepsilon}$, for sufficiently small $\varepsilon>0$, with some constant $C$ satisfies the inequality

$$
\left\|U_{\mathrm{e}}(t, s)\right\| \leq C \text { for } a \leq s \leq t \leq b .
$$

Proof. Let $u=u_{0}(t)$ be completely stable in $[a, b]$ for $\varepsilon \rightarrow 0$ with respect to $(1)_{\varepsilon}$, and $u=u_{\varepsilon}(t)$ and $u=v_{\varepsilon}(t)$ be solutions of $(1)_{\varepsilon}$ such that 
$u_{\varepsilon}(s)=v_{0}(s)$ and $\left\|v_{\varepsilon}(s)-u_{\varepsilon}(s)\right\|<\eta(\delta)$ resp. Then, if $0<\varepsilon<\eta(\delta)$ we must have

$$
\left\|u_{\varepsilon}(t)-v_{\varepsilon}(t)\right\| \leqq\left\|u_{\varepsilon}(t)-u_{0}(t)\right\|+\left\|v_{\varepsilon}(t)-u_{0}(t)\right\|<2 \delta \text { for } \quad s \leq t \leq b .
$$

Hence for any $w \in E$ with $\|w\|<\eta(\delta)$ holds the inequality $\left\|U_{\varepsilon}(t, s) w\right\|$ $<2 \delta$ for $a \leq s \leq t \leq b$. This asserts Lemma 1 .

In order to give our sufficient conditions for the complete stability of a solution, we shall prepare a definition of quasi-regularity of solutions. An $E$-solution $u=u_{0}(t)$ of

$$
\partial_{t} u=A_{0}(t) u+f(t)
$$

is said to be quasi-regular in $[a, b]$ with respect to an operator $A_{1}(t)$, when for any $\delta>0$ there exists an $E$-continuous $v_{\delta}(t)$ on $[a, b]$ such that $\partial_{t} v_{\delta}(t)-A_{0}(t) v_{\delta}(t)-f(t)$ and $A_{1}(t) v_{\delta}(t)$ are bounded and $E$-continuous on $[a, b]$ and the inequalities

$$
\left\|v_{\delta}(t)-u_{0}(t)\right\|<\delta \text { and }\left\|\partial_{t} v_{\delta}(t)-A_{0} v_{\delta}(t)-f(t)\right\|<\delta
$$

hold for $a \leq t \leq b$.

Especially if $A_{0}(t)=A_{1}(t)=A$ and $A$ is the infinitesimal generator of a 1 -parameter semi-group and $f(t)$ is $E$-continuous on $[a, b]$, then an $E$-solution of $(0)$ is quasi-regular in $[a, b]$ with respect to $A$. Indeed in this case we have to set $v_{\delta}(t)=\left(1-\lambda_{\delta}^{-1} A\right)^{-1} u_{0}(t)$ with sufficiently large $\lambda_{\delta}>0$.

Now we assume that $(1)_{\varepsilon}$ is completely $E$-well posed in $[a, b]$ for $\varepsilon \geq 0$ and the operator $A_{\varepsilon}(t)$ have the form :

$$
A_{\varepsilon}(t)=A_{0}(t)+\varepsilon A_{1}(t) \quad(\varepsilon \geq 0) .
$$

Further let $f_{\varepsilon}(t)$ be $E$-continuous on $[a, b]$ and converge to $f_{0}(t)$ uniformly on $[a, b]$ as $\varepsilon \rightarrow 0$. Then we have :

Theorem 2. Let $u=u_{0}(t)$ be an E-solution of $(1)_{0}$ for $\varepsilon=0$ in a finite closed interval $[a, b]$ and be quasi-regular with respect to $A_{1}(t)$ in $[a, b]$. In order that $u=u_{0}(t)$ be completely $E$-stable in $[a, b]$ with respect to $(1)_{\varepsilon}$, it is necessary and sufficient that for sufficiently small $\varepsilon>0$, the fundamental solution $U_{\varepsilon}(t, s)$ of the associated homogeneous equation of $(1)_{\varepsilon}$ is uniformly bounded for $a \leq s \leq t \leq b$.

Proof. As the nessecity of the condition is already given by Lemma 1 , we have only to prove the sufficiency.

For any $\delta>0$ there exists an $E$-continuous $v_{\delta}(t)$ on $[a, b]$ such that 
$h_{\delta}(t)=\partial_{t} v_{\delta}(t)-A_{0}(t) v_{\delta}(t)-f_{0}(t)$ and $A_{1}(t) v_{\delta}(t)$ are $E$-continuous on $[a, b]$ with the conditions

$$
\left\|v_{\delta}(t)-u_{0}(t)\right\|<\delta \text { and }\left\|h_{\delta}(t)\right\|<\delta \text { for } a \leq t \leq b .
$$

Then we get

$$
\partial_{t}\left(u_{\varepsilon}-v_{\delta}\right)=A_{\varepsilon}(t)\left(u_{\varepsilon}-v_{\delta}\right)+\varepsilon A_{1}(t) v_{\delta}+g_{\varepsilon, \delta}(t),
$$

where $g_{\varepsilon, \delta}(t)=f_{\varepsilon}(t)-f_{0}(t)+h_{\delta}(t)$. Hence, by Theorem 1 ,

$$
\begin{aligned}
& u_{\varepsilon}(t)-v_{\delta}(t)=U_{\varepsilon}(t, s)\left\{u_{\varepsilon}(s)-v_{\delta}(s)\right\} \\
& +\int_{s}^{t} U_{\varepsilon}(t, \sigma)\left\{\varepsilon A_{1}(\sigma) v_{\delta}(\sigma)+g_{\varepsilon, \delta}(\sigma)\right\} d \sigma \quad \text { for } \quad a \leq s \leq t \leq b .
\end{aligned}
$$

Thus by (2) we have

$$
\left\|u_{\varepsilon}(t)-v_{\delta}(t)\right\| \leq C\left\{\left\|u_{\varepsilon}(s)-v_{\delta}(s)\right\|+\int_{s}^{t}\left(\varepsilon\left\|A_{1}(\sigma) v_{\delta}(\sigma)\right\|+\left\|g_{\varepsilon, \delta}(\sigma)\right\|\right) d \sigma\right\} .
$$

There exist positive constants $\zeta(\varepsilon)$ and $B_{\delta}$ such that $\zeta(\varepsilon) \rightarrow 0$ as $\varepsilon \rightarrow 0$, $\left\|f_{\varepsilon}(t)-f_{0}(t)\right\|<\zeta(\varepsilon)$ for $a \leq t \leq b$ and $\left\|A_{1}(t) v_{\delta}(t)\right\| \leq B_{\delta}$ for $a \leq t \leq b$. Thus, by (4), we get

$$
\begin{array}{r}
\left\|u_{\varepsilon}(t)-u_{0}(t)\right\| \leq C\left\{\left\|u_{\varepsilon}(s)-u_{0}(s)\right\|+\delta+(b-a)\left(\varepsilon B_{\delta}+\zeta(\varepsilon)+\delta\right)\right\}, \\
\text { for } a \leq s \leq t \leq b .
\end{array}
$$

First taking $\delta>0$ sufficiently small and then letting $\varepsilon \rightarrow 0$, we complete the proof.

REMARK 2. The sufficiency of the condition in Theorem 2 remains valid even for the case of infinite interval $(a, \infty)(b=\infty)$, if we add to it the condition

$$
\int_{a}^{t}\left\|U_{\varepsilon}(t, s)\right\| d s \leq C \text { for } a \leq t<\infty \text { with some constant } C .
$$

\section{$\S 3$. Degeneration of evolutional equations}

Let us consider the evolutional equation of the singular form in the parameter $\varepsilon$ :

$$
\varepsilon \partial_{t} u=A_{\varepsilon}(t) u+f_{\varepsilon}(t) \quad \text { with } \quad \varepsilon>0
$$

and the degenerated equation

$$
A_{0}(t) u+f_{0}(t)=0
$$

We assume that $A_{\varepsilon}(t)$ and $f_{\varepsilon}(t)$ have the forms 


$$
\begin{aligned}
& A_{\varepsilon}(t)=A_{0}(t)+\varepsilon A_{1}(t), \\
& f_{\varepsilon}(t)=f_{0}(t)+\varepsilon f_{1}(t)+\varepsilon h_{\varepsilon}(t),
\end{aligned}
$$

where $f_{0}(t), f_{1}(t)$ and $h_{\mathrm{g}}(t)$ are $E$-continuous on $[a, b]$ and $h_{\mathrm{g}}(t) \rightarrow 0$ uniformly on $[a, b]$ as $\varepsilon \rightarrow 0$.

A solution $u=u_{0}(t)$ of the degenerated equation (1) is said to be completely $E$-stable in $[a, b]$ with respect to $(1)_{\varepsilon}$ for $\varepsilon \rightarrow 0$, when the following condition is fulfilled: For any $\varepsilon>0$ there exists some $\eta(\delta)>0$ such that, if $0<\varepsilon<\eta(\delta)$, any $E$-solution $u_{\varepsilon}(t)$ of $(1)_{\varepsilon}$ in $[s, b]$ for any $s \in[a, b]$ with

$$
\left\|u_{\varepsilon}(s)-u_{0}(s)\right\|<\eta(\delta)
$$

satisfies the inequality

$$
\left\|u_{\mathrm{s}}(t)-u_{\mathrm{o}}(t)\right\|<\delta \text { for } s \leq t \leq b .
$$

Theorem 3. Assume that $(1)_{\varepsilon}$ is completely E-well posed in a finite closed interval $[a, b]$ for $\varepsilon>0$ and $A_{\varepsilon}(t)$ and $f_{\varepsilon}(t)$ have the forms (2). Let $u=u_{0}(t)$ be a E-solution of $(1)_{0}$ on $[a, b]$ such that $u_{0}(t), \partial_{t} u_{0}(t)$ and $A_{1}(t) u_{0}(t)$ are E-continuous on $[a, b]$. In order that $u=u_{0}(t)$ is completely stable in $[a, b]$ for $\varepsilon \rightarrow 0$ with respect to $(1)_{\varepsilon}$ with any E-continuous $f_{1}(t)$ on $[a, b]$, it is necessary and sufficient that the fundamental solution $U_{\varepsilon}(t, s)$ of $\partial_{t} u=\varepsilon^{-1} A_{8}(t) u$ satisfies the following conditions:

1) There exists a constant $C$ such that

$$
\left\|U_{\varepsilon}(t, s)\right\| \leq C \text { for } a \leq s \leq t \leq b \text { and sufficiently small } \varepsilon>0 \text {. }
$$

2) For any $\alpha, \beta, t$, and $v \in E$ such that $a \leq \alpha<\beta \leq t \leq b$,

$$
\int_{\infty}^{\beta} U_{\mathrm{\varepsilon}}(t, s) v d s \rightarrow 0 \text { uniformly on } t \in[\beta, b] \text { as } \varepsilon \rightarrow 0 \text {. }
$$

Proof. The necessity of 1) is obtained in the same way as in the proof of Lemma 1.

To prove the necessity of 2), setting $f_{1}(t)=\phi(t)-A_{1}(t) u_{0}(t)$ with an arbitrary $E$-continuous $\mathcal{p}(t)$ on $[a, b]$, we get from $(1)_{\varepsilon},(1)_{0}$ and (2)

$$
\partial_{t}\left(u_{\mathrm{\varepsilon}}-u_{0}\right)=\varepsilon^{-1} A_{\mathrm{\varepsilon}}(t)\left(u_{\mathrm{\varepsilon}}-u_{0}\right)+\phi(t)+h_{\mathrm{\varepsilon}}(t) .
$$

Hence for $a \leq s \leq t \leq b$

$$
\begin{aligned}
& u_{\varepsilon}(t)-u_{0}(t)=U_{\mathrm{g}}(t, s)\left\{u_{\mathrm{g}}(s)-u_{0}(s)\right\} \\
& +\int_{s}^{t} U_{\varepsilon}(t, \sigma)\left\{\varphi(\sigma)+h_{\mathrm{\varepsilon}}(\sigma)\right\} d \sigma .
\end{aligned}
$$

By 1) we have if $0<\varepsilon<\eta(\delta)$, as $\eta(\delta) \leq \delta$, 


$$
\left\|U_{\varepsilon}(t, s)\left\{u_{\varepsilon}(s)-u_{0}(s)\right\}\right\| \leq C \delta
$$

and

$$
\left\|\int_{s}^{t} U_{\varepsilon}(t, \sigma) h_{\varepsilon}(\sigma) d \sigma\right\| \leq(b-a) C \zeta(\varepsilon)
$$

for $a \leq s \leq t \leq b$, where $\zeta(\delta) \rightarrow 0$ as $\varepsilon \rightarrow 0$. Hence we have only to show that

$$
\text { “ } \int_{s}^{t} U_{\varepsilon}(t, \sigma) \mathcal{P}(\sigma) d \sigma \rightarrow 0 \text { uniformly on } a \leq s \leq t \leq b
$$

as $\varepsilon \rightarrow 0$ for any $E$-continuous $\varphi(t)$ on $[a, b]$ " implies 2). Put $\varphi(\sigma)=\psi(\sigma) v$ with any $v \in E$ and a continuous real valued function $\psi(\sigma)$ on $[a, b]$ such that $0 \leq \psi(\sigma) \leq 1, \psi(\sigma)=1$ for $\alpha+\delta \leq \sigma \leq \beta, \psi(\sigma)=0$ for $a \leq \sigma \leq \alpha$ and for $\beta+\delta \leqq \sigma \leq b$. Then by 1 )

$$
\left\|\int_{s}^{t} U_{\varepsilon}(t, \sigma) \mathcal{\rho}(\sigma) d \sigma-\int_{\infty}^{\beta} U_{\varepsilon}(t, \sigma) v d \sigma\right\| \leq 2 \delta C\|v\| .
$$

Therefore we obtain 2 ), as $\delta$ can be taken arbitrarily small.

To prove the sufficiency of 2 ) with 1 ), we have only to show that by these conditions

$$
\int_{s}^{t} U_{\varepsilon}(t, \sigma) \varphi(\sigma) d \sigma \rightarrow 0 \text { uniformly for } a \leq s \leq t \leq b \text { as } \varepsilon \rightarrow 0 .
$$

Divide the interval $[a, b]$ into a finite number of consecutive intervals $\left[\tau_{\nu-1}, \tau_{\nu}\right](\nu=1, \cdots, N)$ in such a way that $\tau_{\nu}-\tau_{\nu-1}<\delta$ and $\left\|\varphi(\sigma)-\varphi\left(\tau_{\nu}\right)\right\|$ $\left\langle\delta\right.$ for $\sigma \in\left[\tau_{\nu-1}, \tau_{\nu}\right]$. Then

$$
\begin{gathered}
\left\|\int_{s}^{t} U_{\varepsilon}(t, \sigma) \varphi(\sigma) d \sigma-\sum_{s<\tau_{\nu} \leq t} \int_{\tau_{\nu-1}}^{\tau_{\nu}} U_{\mathrm{g}}(t, \sigma) \varphi\left(\tau_{\nu}\right) d \sigma\right\| \\
<C(b-a) \delta+2 C \delta \operatorname{Max}\|\varphi(\sigma)\| .
\end{gathered}
$$

Thus by 2), taking $\delta$ sufficiently small and letting $\varepsilon \rightarrow 0$, we attain to the desired conclusion. Q. E. D.

When $f_{\varepsilon}(t)$ has the form, instead of (2),

$$
f_{\varepsilon}(t)=f_{0}(t)+h_{\varepsilon}(t),
$$

where $f_{0}(t)$ and $h_{\varepsilon}(t)$ have the same meanings as before, we cannot easily have necessary and sufficient conditions for the complete stability of $u_{0}(t)$, but only sufficient conditions, while we can relax the conditions on $u_{0}(t)$ somewhat.

Theorem 4. Let $u=u_{0}(t)$ be an E-continuous solution of $(1)_{0}$ on $[a, b]$ such that for any $\delta>0$ there exists an $E$-continuous $v_{\delta}(t)$ on $[a, b]$ with 
bounded and E-continuous $\partial_{t} v_{\delta}(t), A_{0}(t) v_{\delta}(t)$ and $A_{1}(t) v_{\delta}(t)$ on $[a, b]$ satisfying the conditions

$$
\left\|v_{\delta}(t)-u_{0}(t)\right\|<\delta \quad \text { and } \quad\left\|A_{0} v_{\delta}(t)+f_{0}(t)\right\|<\delta \quad \text { for } t \in[a, b] .
$$

Assume that the fundamental solution $U_{\varepsilon}(t, s)$ of $\partial_{t} u=\varepsilon^{-1} A_{\varepsilon}(t) u$, satisfies for sufficiently small $\varepsilon>0$, the conditions with some constant $C$ :

1) $\left\|U_{\varepsilon}(t, s)\right\| \leq C$ for $a \leq s \leq t \leq b$,

$2)^{\prime} \quad \int_{a}^{t}\left\|U_{\varepsilon}(t, s)\right\| d s \leq \varepsilon C$ for $a \leq t \leq b$.

Then $u=u_{0}(t)$ is completely $E$-stable in $[a, b]$ with respect to $(1)_{\varepsilon}$ for $\varepsilon \rightarrow 0$.

Proof will be left to the reader.

REMARK 3. The sufficiency of the conditions 1 ) and 2$)^{\prime}$ in Theorem 4 remains valid even for the case of infinite interval $(a, \infty)(b=\infty)$.

\section{§4. Degeneration of evolutional equation when $A_{\varepsilon}(t)=A$}

Consider the evolutional equation of singular form in $\varepsilon$ :

$$
\varepsilon \partial_{t} u=A u+f_{\varepsilon}(t) \quad(\varepsilon>0),
$$

where $A$ is the infinitesimal generator of a one parameter semi-group in a reflexive Banach space $E$. As it has been stated in $\S 1$, the operator $A$ can be extended to a continuous linear operator on $E$ into $E_{1}$, and the equation $(1)_{\varepsilon}$ becomes completely $E$-well posed in any finite interval. The fundamental solution of the associated homogeneous equation has the form

$$
U_{\varepsilon}(t, s)=\exp \left(\varepsilon^{-1}(t-s) A\right),
$$

as $\exp (t A)(t \geqq 0)$ is the transformation generated by $A$.

Theorem 5. Let $u=u_{0}(t)$ be an E-solution in a finite closed interval $[a, b]$ of the degenerated equation

$$
A u+f_{0}(t)=0
$$

with E-continuous $\partial_{t} u_{0}(t)$ on $[a, b]$.

In order that $u_{0}(t)$ be completely E-stable in $[a, b]$ with respect to $(1)_{\varepsilon}$ for $\varepsilon \rightarrow 0$, where $f_{\varepsilon}$ has the form (2) with any E-continuous $f_{1}$, it is necessary and sufficient that the following conditions are fulfilled:

1) With some constant $C,\|\exp (t A)\| \leq C$ for $0 \leq t<\infty$.

2) $A v=0$ with $v \in \mathscr{D}(A)$ implies $v=0$, 
where $\mathscr{D}(A)$ denotes the proper domain of $A$ before the extension of $A$ on $E$.

Proof. From Theorem 3 we get easily the necessity of 1), as $U_{\varepsilon}(t, s)=\exp \left(\varepsilon^{-1}(t-s) A\right)$.

By the mean ergodic theorem in a reflexive Banach space, we obtain from 1) a projective operator $P$ on $E$ into $E$ such that:

$$
\begin{gathered}
\lim _{\tau \rightarrow \infty} \tau^{-1} \int_{0}^{\tau} \exp (t A) v d t=P v \quad \text { for any } v \in E,{ }^{2)} \\
P \exp (t A)=\exp (t A) P=P^{2}=P,
\end{gathered}
$$

and

$$
P(E)=\{u \in \mathscr{D}(A) ; A u=0\}
$$

Thus, for $a \leq \alpha<\beta \leq t \leq b$ and any $v \in E$, setting

$$
\begin{aligned}
& \tau(\varepsilon)=\varepsilon^{-1}(\beta-\alpha) \text { we have by }(4) \\
& \int_{\alpha}^{\beta} U_{\varepsilon}(t, s) v d s-(\beta-\alpha) P v \\
& \quad=\int_{\alpha}^{\beta} \exp \left(\varepsilon^{-1}(t-s) A\right) v d s-(\beta-\alpha) P v \\
& \quad=(\beta-\alpha) \exp \left(\varepsilon^{-1}(t-\beta) A\right)\left\{\tau(\varepsilon)^{-1} \int_{0}^{\tau} \exp (\sigma A) v d \sigma-P v\right\},
\end{aligned}
$$

hence by (3) and 1), we have

$$
\left\|\int_{\infty}^{\beta} U_{\varepsilon}(t, s) v d s-(\beta-\alpha) P v\right\| \rightarrow 0 \text { uniformly for } \beta \leq t \leq b,
$$

as $\tau(\varepsilon) \rightarrow \infty$ for $\varepsilon \rightarrow 0$.

Hence the condition 2) in Theorem 3 with 1) is equivalent to:

$$
P v=0 \text { for every } v \in E .
$$

Therefore, by (5) the condition 2) with 1) is equivalent to the condition 2) with 1) in Theorem 3. Q. E. D.

REMARK 4. The sufficiency of the conditions in Theorem 5 remains valid even for the case $b=\infty$, if we replace 2) by

$$
\int_{0}^{\infty}\|\exp (t A)\| d t<\infty \text {. }
$$

2) Here the lim means the strong limit in $E$. 


\section{OSAKA UNIVERSITY}

(Received March 5, 1963)

\section{References}

[1] M. Nagumo: Re-topologization of functional space in order that a set of operators will be continuous, Proc. Japan Acad. 37 (1961), 550-552.

[2] M. Nagumo: Singular perturbation of Cauchy problem of partial differential equations with constant coefficients, Proc. Japan Acad. 35 (1959), 449-454.

[3] H. Kumano-go: Singular perturbation of Cauchy problem of partial differential equations with contant coefficients II, Proc. Japan Acad. 35 (1959), 541-546.

[4] K. Yosida: Mean ergodic theorem in Banach spaces, Proc. Imp. Acad. Japan (1938), 292-294.

[5] E. Hille and R. S. Phillips: Functional analysis and semi-groups, Amer. Math. Soc. Colloq. Publ. 31, Providence, 1957. 